\title{
Los programas de investigación, promoción e intervención social para la mejora de habilidfades de lectocomprensión en la Región Ameca Jalisco en CUValles, Universidad de Guadalajara
}

DOI: https://doi.org/10.32870/dse.v0i11.274

\section{Mónica Almeida López* \\ Rachel García Reynaga** \\ Hilde Eliazer Aquino López*** \\ María Teresa Orozco López**** \\ Colaborador: Pablo Silva Villalever $* * * * *$ \\ Colaborador: Víctor Montero Gaytán******}

Resumen: Este trabajo presenta los resultados obtenidos con la metodología de investigación-acción en la mejora de las habilidades de lectocomprensión en el contexto de la Región Ameca, compuesta por 16 municipios. Ahí, universitarios-capacitadores del programa endógeno del CUValles atienden a alumnos de educación básica y media superior, con la finalidad de retroalimentarse en su conocimiento lecto-comprehensivo al transmitir la educación hacia su comunidad, lo que también resulta un beneficio social. Este planteamiento de ida y vuelta es resultado de los programas nombrados Expresa y Sale y Valee. Su fundamento contiene el concepto "alfabetismo" o "literacy" en habla inglesa, que en síntesis son formas socialmente reconocidas de generar, comunicar y negociar contenidos significativos; es decir, una plataforma del conocer en cuatro ejes principales para el desarrollo universitario y comunal: lenguaje materno, lengua extranjera angloparlante, lenguaje tecnológico computacional e informático y lenguaje matemático. Palabras clave: Investigación-acción, Expresa, Sale y Valee, alfabetismos, lectocomprensión.

\begin{abstract}
This work shows the results obtained with the action research methodology to develop the skills of read comprehension on Ameca district of Jalisco State, constituted with 16 localities examined; a lot of middle school students were evaluated with overseers from CUValles, Universidad of Guadalajara. The purpose of this way is to get feed-back knowledge by the university students; otherwise also those actions could be translated to communitarian benefits. In fact, this work contained two local programs called Expresa and Sale y Valee within the students learn literacy abilities and teach to read at the community respectively. The main reason in the text is the concept of "alfabetismo" or literacy, there are both, forms of basically skills or knowledge of a particular subject with meaning; in this case we studied four blocks of "alfabetismos": native language, foreign language (English), technology-informatics language and mathematic language. Keywords: Action Research, read comprehension skills, "Alfabetismos", Literacy.
\end{abstract}

* Doctorado en análisis, representación y ordenación del territorio de la Universidad de Oviedo en línea de cooperación y bienestar social. Profesora de tiempo completo del Sistema de Universidad Virtual de la Universidad de Guadalajara. Correo electrónico: Almeida@redudg.udg.mx

**Doctorado en Educación con especialidad en metodología de la enseñanza. Profesora de tiempo completo del Centro Universitario de los Valles de la Universidad de Guadalajara. Correo electrónico: rachel.garcia@,valles.udg.mx

***Doctorado en Educación por la Universidad La Salle, programa interinstitucional. Profesora investigadora medio tiempo en Unidad 141 de la Universidad Pedagógica Nacional. Correo electrónico: heliazer@gmail.com

****Doctorado en Humanidades y Artes en la Universidad Autónoma de Zacatecas con línea en lectura y literatura infantil y juvenil. Directora de la Escuela Normal para Educadoras de Guadalajara. Correo electrónico: Mariateresa.orozco@jalisco.gob.mx.

*****Maestrante en Tecnologías para el aprendizaje de la Universidad de Guadalajara. Profesor de Asignatura "A" en la carrera de psicología CUValles. Correo electrónico: pvillalever1@yahoo.com

******Profesor del Centro Universitario de los Valles. Estudiante del Doctorado en Gestión de la educación Superior. Universidad de Guadalajara. Correo electrónico: victor.montero@,valles.udg.mx 
Mónica Almeida López, Rachel Garcíaeres, Reynaga, Hilde Aquino, Teresa Orozco. Colaboradores: Pablo

Silva Villalever y Víctor Montero Gaytán

\section{Presentación}

En los albores del siglo XXI, las actividades de educación y formación en todos sus aspectos se han convertido en los principales motores del desarrollo. También contribuyen al progreso científico y tecnológico, y al avance general del conocimiento que constituye el factor más decisivo del crecimiento económico.

La educación es el eje transformador de las sociedades modernas y sus cualidades pueden contribuir en la construcción de una comunidad global y local que ofrezca mejores condiciones de vida tanto en el aspecto material, en las diversas manifestaciones culturales y en las formas de convivencia en un ambiente de libertad, certidumbre y esperanza.

Por tanto es menester modelar un sistema educativo que atienda de manera eficaz las necesidades de desarrollo socio-cognitivo que exigen cada una de las etapas de la vida de los estudiantes, al reconocer sus potencialidades y sus limitaciones como individuos que por su naturaleza biológica y social son diferentes; como tal, es preponderante adecuar la teoría a la realidad, creando un modelo pedagógico plurivalente que permita orientar esa gama humana hacia la autoconciencia; es decir, ser capaz de llegar a la autosatisfacción personal en el sentido más amplio, a la par de producir cambios en su entorno con beneficio social.

Para que un sistema educativo tenga éxito es necesario que sus acciones tengan como punto de partida un modelo educativo que considere, entre otras variables, las capacidades de los profesores, las herramientas y espacios que utilizan, las condiciones socioeconómicas, motivacionales y académicas de los estudiantes, así como las habilidades que se desean desarrollar y los conocimientos que se deben adquirir.

Históricamente la educación se ha entendido como un fenómeno social. De Ibarrola (1985) señala a Durkheim como uno de los principales teóricos del aspecto social de la educación, al establecer la naturaleza social del fenómeno educativo, además de la relación existente entre los cambios de las estructuras sociales y las transformaciones de los sistemas de educación.

Los planteamientos de Durkheim coinciden en definir a la educación como la acción ejercida por las generaciones adultas sobre las generaciones todavía no maduras para la vida social, destacando que su objetivo es despertar y desarrollar en la persona aquellas actitudes físicas, intelectuales y morales que le exige la sociedad en general y el medio al cual está especialmente destinado (De Ibarrola, 1985: 253).

Actualmente no queda duda del vínculo existente entre sociedad y educación, y de cómo los cambios educativos deben ir al unísono de las transformaciones sociales, culturales, políticas y también económicas. La educación, pues, resulta importante para las sociedades puesto que en ella se sustentan muchos de los fundamentos de los que se servirán al enfrentarse a los diversos retos del avance social, incluyendo el sistema educativo de cada país, sus costumbres, identidades e ideologías, entre otros aspectos propios de su historia y del momento determinado de su desarrollo, lo que dará paso a cambios paradigmáticos en la forma de concebir al mundo. 
Los programas de investigación, promoción e intervención social para la mejora de habilidfades de lectocomprensión en la Región Ameca Jalisco en CUValles, Universidad de Guadalajara

Estamos convencidos de que la educación superior debería estar plenamente vinculada al sistema de educación básica, porque a fin de cuentas los estudiantes que se atenderán en las aulas universitarias son el resultado de las prácticas de enseñanza-aprendizaje que se llevan a cabo en la educación básica y media superior; debe ser un mismo proceso para el desarrollo de nuestra sociedad mexicana. Asimismo, la investigación que se desarrolla sobre la educación debe impactar en la enseñanza de todos los niveles.

La educación contribuye al desarrollo humano en todos sus aspectos. Como condición previa se tiene que la educación básica para todos es vital. En la medida en que el desarrollo tiene como objeto la plena realización del ser humano como tal, y no como medio de producción, es claro que esa educación básica deberá abarcar todos los elementos del saber necesarios para acceder eventualmente a otros niveles de formación. Sin embargo, en la perspectiva de la educación permanente, la educación básica deberá además y sobre todo dar a cada persona los medios de modelar libremente su vida y participar en la evolución de la sociedad (Delors, 1996: 82).

De acuerdo con Morín (1999) la educación tendría que preocuparse por crear cabezas bien puestas más que bien llenas, entendiendo por pensar "bien" la habilidad de contextualizar y aplicar los saberes incorporados a la solución de problemas específicos de su entorno. Morín (2002) propone siete puntos de vista a considerar en la educación: que en forma constante se luche contra las cegueras del conocimiento: el error y la ilusión. Que se enseñen los principios de un conocimiento pertinente que contemple "el contexto", "lo global", "lo multidimensional" y "lo complejo". La educación tiene que promover una inteligencia general. Que se enseñe la condición humana: por su naturaleza, el ser humano es a la vez físico, biológico, psíquico, cultural, social e histórico. Que se asuma y enseñe la identidad terrenal. Que se eduque para enfrentar la incertidumbre. Que se enseñe la comprensión como medio y fin de la comunicación humana. Que se enseñe la ética del ser humano.

Maturana afirma que no nacemos humanos, nos hacemos humanos en la interacción con otros seres humanos. La educación es una transformación en la convivencia, en la que uno no aprende una temática sino que aprende un vivir y un convivir. Se es humano no desde la genética sino desde la convivencia.

Este marco complejo implica considerar al ser humano en toda su diversidad, como individuo biológico, social, cultural, afectivo. Estamos conscientes de que precisamente el olvido de la plenitud del ser humano, así como la mirada fragmentada de la realidad, ha llevado a que prevalezca unilateralmente el desarrollo técnico y económico a costa de la calidad de vida de los individuos, lo que ha contribuido a que se agudicen los problemas de iniquidad y abuso en la sociedad.

El mundo podría asumirse como series de sistemas de organización biológica o social semicerrados que se encuentran en constante dinámica. Siguiendo a Maturana, sistemas que son autopoiéticos, esto es, con la capacidad de auto reformarse, reproducirse y autorregularse (Maturana y Varela, 1990). 
El aprendizaje está mediado por el lenguaje y éste es el mediador del pensamiento, la imaginación, la memoria, la percepción y demás operaciones de orden superior necesarias para el manejo de diversos dominios del conocimiento científico, artístico, tecnológico, etcétera.

La finalidad de cualquier proyecto educativo no debe ser sólo la acumulación de información, sino el desarrollo de habilidades para aprender y para saber aplicar el conocimiento en situaciones de vida.

El lenguaje es un fenómeno social y por ende un producto cultural. Todas las lenguas son sistemas de símbolos con reglas convencionales socialmente establecidas. Tradicionalmente se asocia el lenguaje con su forma escrita, de lectura y escritura, y se demeritan las actividades de escuchar y hablar como parte de la misma competencia comunicativa.

El lenguaje permite al ser humano representar objetos y acciones, entender y discutir ideas abstractas, inventar historias e intercambiar información compleja. En gran medida, los conocimientos que construimos los seres humanos en relación con el mundo son a partir del lenguaje. El lenguaje es la materia, el medio y el horizonte de toda vida mental, de toda vida social y de toda vida individual.

La sociedad de la información requiere el manejo de diversos lenguajes de manera simultánea. No basta con manejar y dominar el idioma que se aprende de manera natural; los requerimientos del mundo globalizado exigen, además, el manejo de un lengua extranjera y de la potenciación de otros lenguajes en la vida cotidiana como el lógico-matemático y el digital, lo que se conoce como alfabetismos. Se utiliza el término "alfabetismos" para referirnos a los saberes básicos, esenciales o claves que la institución escolar debe favorecer en los estudiantes. Hablar de alfabetismos refiere a la necesidad de comunicación a través de distintos lenguajes, no sólo el lenguaje oral y escrito.

Los alfabetismos se definen como "formas socialmente reconocidas de generar, comunicar y negociar contenidos significativos por medio de textos codificados en contextos de participación en Discursos o como miembros de Discurso" (Lankshear y Knobel, 2008: 74).

El concepto de "nuevos alfabetismos" se sitúa como centro de la política educativa en el ámbito mundial del desarrollo curricular y el pensamiento cotidiano sobre la práctica educativa (Lankshear y Knobel, 2008) Los nuevos alfabetismos expanden la metáfora de la lectura y la escritura a un "paisaje textual" en donde el aprendizaje de la lectoescritura en lengua materna y extranjera, del pensamiento lógico matemático y de las nuevas tecnologías de información y comunicación (TIC) hacen referencia a la capacidad de leer y escribir distintos tipos de textos, signos, artefactos, matices e imágenes por medio de las cuales nos vinculamos y comprometemos con la sociedad en un sentido amplio (Lankshear y Snyder, 2000).

Freire establece que "La educación, cualquiera que sea el nivel en que se dé, se hará tanto más verdadera cuanto más estimule el desarrollo de esa necesidad radical de los seres humanos, la de su expresividad" (Freire, 2001: 54). Desde esta postura el término "alfabetismo" rebasa su significado referido a la capacidad para leer y escribir para referirse a la capacidad para apropiarse de la información con independencia de cómo se presente. 
Los programas de investigación, promoción e intervención social para la mejora de habilidfades de lectocomprensión en la Región Ameca Jalisco en CUValles, Universidad de Guadalajara

La pregunta central en el tema de los alfabetismos es cómo se puede adquirir y desarrollar la competencia para leer la realidad y comunicarla. El alfabeto es uno de los principales recursos, pero a él hay que añadir el lógico matemático y el digital entre otros. Para saber cómo desarrollar la competencia interpretativa se deben considerar las prácticas retóricas que se aplican. Y por prácticas retóricas entendemos los entornos comunicativos y los procesos de negociación de significados.

El término "alfabetismo" tiene su raíz en el término "alfabetización" el cual fue utilizado por Freire para referirse a la necesidad de aprender a leer (en el sentido de saber decodificar) pero también en el sentido de aprender a leer la realidad. Freire establece que:

Hablar de alfabetización de adultos y de bibliotecas populares es hablar, entre muchos otros, del problema de la lectura y la escritura. No de la lectura de palabras y de su escritura en sí mismas, como si leerlas y escribirlas no implicara otra lectura, previa y concomitante a aquélla, la lectura de la realidad misma.

La comprensión crítica de la alfabetización que incluye la comprensión igualmente crítica de la lectura, exige la comprensión crítica de la biblioteca (Freire, 2001: 108).

En el contexto de la sociedad de la información el término alfabetismo se utiliza como opción de traducción castellana de "literacy" término que propone el Organismo para la Cooperación y el Desarrollo Económico (OECD por sus siglas en inglés), que ha sido traducido en el ámbito hispanoparlante como cultura, formación, alfabetización, aptitud, habilidad, incluso "literacidad", pero que en México se ha traducido como competencia.

El Programa para la Evaluación Internacional de Alumnos de la OECD (PISA, por sus siglas en inglés) establece:

PISA se centra en la capacidad de los estudiantes para usar los conocimientos y habilidades y no en saber hasta qué punto dominan un plan de estudios o currículo escolar.

Por ello, no mide qué tanto pueden reproducir lo que han aprendido, sino que indaga lo que en PISA se denomina competencia (literacy); es decir, la capacidad de extrapolar lo que se ha aprendido a lo largo de la vida y su aplicación en situaciones del mundo real, así como la capacidad de analizar, razonar y comunicar con eficacia al plantear, interpretar y resolver problemas en una amplia variedad de situaciones (INEE, 2009: 13-14).

En el informe sobre los resultados de la aplicación de esta prueba a México en 2009 se establece: El concepto de literacy o competencia se refiere a la capacidad de los estudiantes para extrapolar lo que han aprendido, y aplicar sus conocimientos y habilidades en nuevos escenarios, así como para analizar, razonar y comunicarse de manera satisfactoria al plantear, resolver e interpretar problemas en diversas situaciones del mundo real.

En este enfoque, la adquisición de competencias es un proceso que dura toda la vida y no sólo se obtiene a través de la escuela o el aprendizaje formal, sino mediante la interacción con los compañeros, los pares y la sociedad. Las competencias también se identifican con habilidades complejas que son relevantes para el bienestar personal, social y económico en la vida como adultos (INEE, 2009: 20). 
El uso del término parte de una perspectiva sociocultural en la cual es imposible separar las prácticas sociales mediadas por los textos de los elementos relacionados con la lectura y la escritura o cualquier otro sentido de aplicación del término. Para tratarlos independientemente de los elementos no impresos como los valores, el contexto o el significado: "Los elementos del alfabetismo no existen aparte de las prácticas sociales en las que se incluyen y en las que se adquieren" (Gee, 1996: 28).

Existe un término utilizado para referirse a las nuevas prácticas comunicativas de los jóvenes en la sociedad del conocimiento. Se llama: new literacys. Este término es utilizado por Lankshear, quien sitúa el alfabetismo como centro de la política educativa a nivel mundial.

Las alfabetizaciones son ahora el elemento central de la política de educación, del desarrollo curricular y el pensamiento cotidiano sobre la práctica educativa. Es difícil creer que, hace sólo dos o tres decenios, el término "alfabetización" casi no aparecía en el discurso educativo formal. En cambio, existía un campo de rancio abolengo conocido como "lectura" (Lankshear y Knobel, 2008: 23).

Lanham (1995: 198) afirma que el "alfabetismo" ha ampliado su alcance semántico desde "la capacidad de leer y escribir" al significado actual de "la capacidad de comprender la información con independencia de cómo se presente (Lankshear y Knobel, 2008: 36).

Resulta prioritario el desarrollo de programas que posibiliten una intervención en un doble sentido: 1) que forme críticamente a los participantes, en este contexto los estudiantes de licenciatura, en habilidades comunicativas y 2) que se les habilite para el desarrollo de un programa comunitario en el que desarrollen sus competencias para la solución de problemas que se les presenten tanto en el área académica como en su comunidad.

El proyecto socioeducativo mencionado es planeado y propuesto pensándolo en relación con un contexto social histórico y geográfico específico en la Región Valles del estado de Jalisco, México; el cual es condicionado por factores sociales, culturales, económicos y políticos que se toman en consideración en su planeación, desarrollo y evaluación.

Atendiendo el marco de los estatutos que la Universidad de Guadalajara en su Plan de Desarrollo Institucional visión 2030, establece como una de sus políticas en la línea estratégica de formación y docencia: "Promover un modelo educativo enfocado en el estudiante y centrado en el aprendizaje, apoyado en las mejores prácticas pedagógicas y en las tecnologías de la información y la comunicación" (UdeG, 2014: 19).

En octubre de 2012, en el CUValles, en Consejo de Centro se aprobó el programa "Potenciación de Alfabetismos" y contempla la potenciación de cuatro alfabetismos fundamentales en lengua materna, lengua extranjera, lenguaje lógico matemático y lenguaje digital.

El Plan de Desarrollo del Centro Universitario de los Valles 2030 segunda actualización, contempla entre sus objetivos centrales el fortalecimiento de los perfiles profesionales con acciones curriculares actualizadas y pertinentes; desarrollar el conocimiento y dominio de los cuatro alfabe- 
Los programas de investigación, promoción e intervención social para la mejora de habilidfades de lectocomprensión en la Región Ameca Jalisco en CUValles, Universidad de Guadalajara

tismos antes mencionados. Lo anterior con el fin que los estudiantes adquieran los conocimientos y las habilidades de liderazgo que les permitan entender en su justa dimensión los problemas de sus comunidades y de la región, además de la capacidad para encontrar los canales gubernamentales y sociales que puedan ofrecer recursos materiales y humanos para su solución.

El concepto de alfabetismo (literacy) alude a una herramienta útil para adquirir y comunicar información. Este concepto es cercano a la noción de competencia lectora propuesta en PISA, la cual refiere a un proceso de aplicación activo, deliberado y funcional de lectura (INEE, 2009: 44).

La imagen de lectura en la sociedad actual se basa en la decodificación del código escrito. Sin embargo, el conocimiento y la decodificación de un idioma no implican comprensión. La comprensión es uno de los niveles de lectura. PISA establece que "La competencia lectora abarca una amplia variedad de habilidades cognitivas como la decodificación, el conocimiento de las palabras, la gramática y las estructuras y características lingüísticas y textuales, así como el conocimiento del mundo. Además, incluye aspectos de índole metacognitiva como la conciencia en la habilidad de usar diferentes estrategias que sean apropiadas al leer los textos" (INEE, 2009: 43).

La competencia lectora no se considera una capacidad que se adquiere sólo en la infancia, durante los primeros años de escolarización, se concibe como un proceso en evolución, el cual incluye una serie de conocimientos, habilidades y estrategias que las personas van construyendo a lo largo y ancho de la vida en ámbitos diversos, mediante la interacción con los compañeros, la familia y con la sociedad en la que están inmersos.

Las capacidades lingüísticas se perfeccionan usando el lenguaje en contextos significativos, así como los niños aprenden a hablar sin instrucción directa y en un lapso relativamente breve porque quieren interactuar con otros y expresar sus sentimientos y deseos, también la comunicación ha de ser la fuerza que impulse a los seres humanos para continuar en el encuentro significativo de la lectura y la escritura.

El enfoque comunicativo del lenguaje (escuchar, hablar, leer y escribir) permite interpretar el mundo. Permite al ser humano representar objetos y acciones, entender y discutir ideas abstractas, inventar historias e intercambiar información compleja.

Actualmente, la información del mundo que nos rodea llega no solamente en palabras escritas sobre papel sino, cada vez con mayor frecuencia, mediante imágenes y sonidos. El campo del alfabetismo ha surgido en la educación con el objeto de organizar y promover la importancia que tiene enseñar esta noción ampliada de alfabetismo. En el centro mismo de ella se encuentran las habilidades de pensamiento crítico de orden superior, por ejemplo: saber cómo identificar conceptos claves, cómo establecer conexiones entre una multitud de ideas, cómo formular preguntas pertinentes, elaborar respuestas e identificar falacias, entre otros, que conforman el verdadero cimiento tanto de la libertad intelectual como del ejercicio pleno de la ciudadanía dentro de una sociedad democrática.

Los textos literarios cumplen una doble función: muestran una realidad histórica y facilitan estrategias para que el lector recree los motivos y condicionantes que originaron dicha creación. 
La pervivencia de la obra literaria se debe a que se interpreta a la luz de una cultura determinada; es decir, es "reescrita" por cada una de las sociedades que la leen y por cada uno de los lectores.

La interacción con textos literarios favorece la competencia comunicativa que le lleva a producir su propio discurso. Con esta perspectiva comunicativa y funcional se favorece el desarrollo de las capacidades de comprensión y expresión de los mensajes orales y escritos, con diferentes intenciones, utilizando códigos lingüísticos y no lingüísticos. Facilita al alumnado la construcción de su propio pensamiento.

Con base en los bajos resultados de México en evaluaciones internacionales (prueba PISA) en los campos de comprensión lectora y de razonamiento lógico-matemático, aunado a los bajos puntajes de ingreso a la Universidad de Guadalajara de los alumnos en CUValles, el proyecto de talleres de formación integral se diversificó y fortaleció a través de cursos y talleres para conformar una oferta de fortalecimiento de las capacidades lectoras, fundamentales para el resto de las habilidades y el desarrollo del pensamiento científico de los estudiantes de CUValles, las cuales apoyen y garanticen una trayectoria universitaria exitosa.

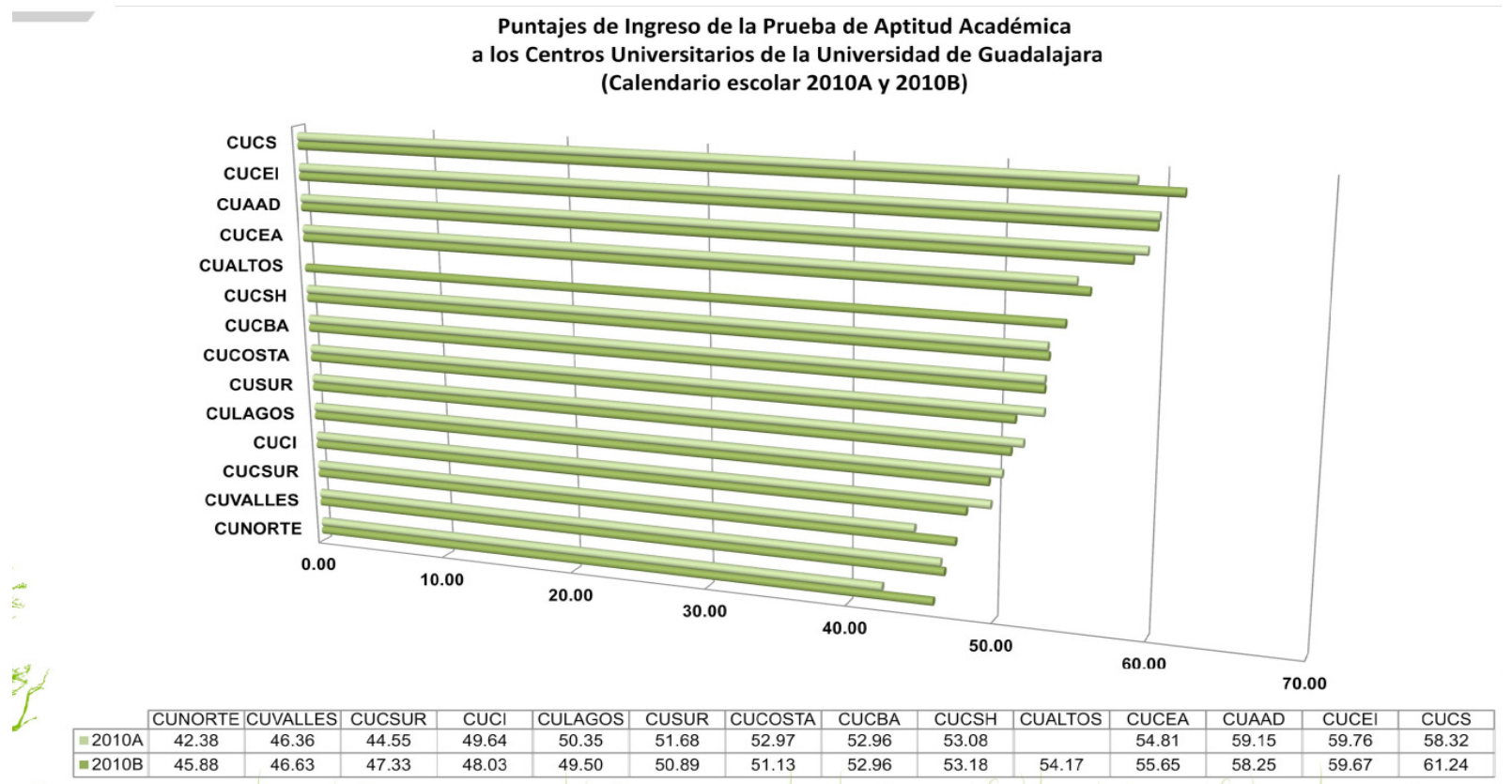

FUENTE: Elaborada con datos proporcionados por la Coordinación General de Control Escolar de la Universidad de Guadalajara.

En la parte correspondiente a la potenciación del alfabetismo en lengua materna se desarrolló el programa denominado Expresa-Sale y Valee, el cual se atendió en un primer momento a través de talleres de formación lectora en donde los asistentes realizaron un encuentro lúdico y placentero con los libros y la lectura con el propósito de construir una red de lectores críticos tanto al interior como al exterior de CUValles. 
Los programas de investigación, promoción e intervención social para la mejora de habilidfades de lectocomprensión en la Región Ameca Jalisco en CUValles, Universidad de Guadalajara

El programa tiene dos vertientes, una al interior llamada Expresa y otra al exterior denominada Sale y Valee. En ambas vertientes la metodología es similar, la diferencia es que en Expresa se forma a los estudiantes del CUValles para convertirlos en lectores y promotores sociales y en Sale y Valee el nuevo promotor social comparte su experiencia con los estudiantes de educación básica, bachillerato y población abierta de la Región de los Valles.

El programa consta de seis fases, a cubrirse en tres años, denominadas cada una de ellas: interpreta, interpreta-dos, comunica, comunica-dos, comparte y comparti-dos.

El trayecto formativo Expresa al interior del CUValles contempla un total de 360 horas de formación y cada una de las fases se opera bajo la modalidad teórico-práctica con distintas horas, pudiendo cubrirse en tres años. Al terminar los estudiantes, ahora como promotores sociales, se incorporan en la intervención social a través de Sale y Valee, éste con un total de 480 horas validadas como servicio social.

Expresa tiene como propósito la formación de lectores eficientes y críticos a través un encuentro lúdico y placentero con los libros y la lectura a partir de potenciar la habilidad en la lengua materna, base del pensamiento y esencial para los procesos de aprendizaje.

Este programa fomenta la reflexión, análisis e interpretación de textos con el propósito de potenciar el dominio del idioma español que permita a los estudiantes representar objetos y acciones, entender y discutir ideas abstractas, inventar historias, motivar la imaginación y creatividad además de poder intercambiar información compleja en diferentes situaciones y contextos a partir de tres ejes:

a) Interpretar: acciones encaminadas a encontrar el sentido de cualquier tipo de texto.

b) Argumentar: comprende aquellas acciones relacionadas con la justificación o sustentación de la información, la explicación del porqué de las proposiciones y la sustentación de las conclusiones.

c) Proponer: se refleja en acciones como el planteamiento de soluciones a conflictos de tipo social, la generación de hipótesis y la construcción de mundos posibles derivados de la interpretación de las lecturas y su posterior reescritura.

Haciendo un comparativo, PISA establece tres habilidades a desarrollar en relación con la lectura: a) Acceder y recuperar. Implica habilidades asociadas a buscar, seleccionar y reunir información. En algunas ocasiones los lectores buscan una información específica en un texto, por ejemplo, el nombre del autor, el horario de las películas, el teléfono de una empresa, etcétera. También pueden necesitar localizar un dato o un hecho que confirme o rectifique la afirmación realizada por alguna persona. Para lograr su cometido, los lectores acceden a un espacio de información (por ejemplo la página de un libro, una tabla o una lista) en donde se ubican los datos que necesitan. Recorren ese 
Mónica Almeida López, Rachel Garcíaeres, Reynaga, Hilde Aquino, Teresa Orozco. Colaboradores: Pablo Silva Villalever y Víctor Montero Gaytán

espacio en búsqueda de la información requerida hasta encontrarla, la seleccionan y finalmente la obtienen. Con el término acceder se describe, precisamente, a este proceso de ingresar a ese espacio en donde se localiza la información, y con recuperar al de seleccionar la información necesaria y obtenerla.

b) Integrar e interpretar. Este proceso se refiere al procesamiento de lo que se lee para darle un sentido propio. Integrar requiere que el lector comprenda la relación entre diferentes partes, así como demostrar y entender la coherencia de un texto; es decir, cuando se integra se reconoce esta coherencia tanto en lo particular como en lo general. Un estudiante deberá distinguir, por ejemplo, cuál es la relación que se establece entre dos oraciones, entre varios párrafos o entre distintos textos. En cada caso, este proceso implica relacionar varias piezas de información para darle sentido, y así saber si estas informaciones son iguales o diferentes, si son comparables o si guardan una relación de causalidad. Interpretar hace referencia al proceso de darle sentido a algo que no está completamente determinado. Cuando se interpreta, un lector identifica las suposiciones o implicaciones que subyacen en una parte o en todo el texto. Por ejemplo, se puede solicitar al lector inferir la relación establecida entre dos partes del texto, distinguir las ideas principales de las secundarias, o que encuentre un caso específico de algo descrito de forma general.

c) Reflexionar y evaluar. Implica aprovechar el conocimiento, las ideas o valores que están más allá del texto con el propósito de relacionar la información dada dentro de éste con los propios marcos de referencia del lector, ya sea conceptual o basados en su experiencia. En la reflexión se busca que los lectores utilicen su propio conocimiento y experiencia para comparar, contrastar o formular hipótesis. En la evaluación deben realizar un juicio acerca del texto, empleando referencias como la experiencia personal o el conocimiento formal.

\begin{tabular}{|l|l|l|}
\hline $\begin{array}{l}\text { Habilidades que con- } \\
\text { templa PISA }\end{array}$ & Énfasis del programa & Fases Expresa \\
\hline Acceder y recuperar & Interpretar (Lectura literaria) & Interpreta/Interpretados \\
\hline Integrar e interpretar & Argumentar (Expresión comunicativa) & Comunica/Comunicados \\
\hline Reflexionar y evaluar & Proponer (Transposición) & Comparte/Compartidos \\
\hline & Servicio social & Sale y Valee \\
\hline
\end{tabular}

El marco teórico del programa se sustentó en considerar al alumno con base en edades lectoras y no en edades cronológicas para la definición de los niveles de comprensión lectora y tipo de libros que le eran promovidos a leer, ya que dar por sentado que los años escolarizados de educación básica y bachillerato garantizan una formación lectora eficiente sería continuar con la dinámica de simulación en donde lo importante es sólo el conocimiento del código lingüístico y su decodificación. 
Los programas de investigación, promoción e intervención social para la mejora de habilidfades de lectocomprensión en la Región Ameca Jalisco en CUValles, Universidad de Guadalajara

Sobre la base de la metodología de la investigación acción, la cual está orientada al cambio educativo construido desde y para la práctica a través de su transformación con la participación de los propios sujetos implicados en su propia mejora y en colaboración durante el proceso, la propuesta se estructuró con dos vertientes: al interior (endógena) del Centro Universitario con talleres semanales Expresa llevados a los estudiantes de nivel superior del Centro Universitario; y al exterior (exógena) del Centro Universitario con intervenciones en estudiantes de escuelas públicas de nivel básico de los distintos municipios que conforman la región de los Valles, programa Sale y Valee, al asistir una vez a la semana a trabajar la lectura literaria de cuentos con los alumnos y transponer lo vivenciado en los talleres internos.

\section{Expresa (vertiente interna)}

La vertiente interna es conocida como Expresa, en ella se busca la formación de lectores dentro del CUValles, en esta fase los programas educativos introducen en su malla curricular los talleres de formación en el área de lectura literaria y bases pedagógicas. El programa consta de seis fases:

\section{a) Fase I y II, talleres de lectura literaria (Interpreta e Interpretados):}

El propósito no fue atender los niveles de comprensión lectora, sino trabajar la formación de lectores a través de la lectura literaria, de manera simultánea a las acciones orientadas a la promoción de la lectura como Libros Libres; esto por medio de acercamientos lúdicos libres y en la medida de lo posible fuera del contexto áulico en torno al libro y la lectura.

b) Fase III y IV, talleres de expresión comunicativa (Comunica y Comunicados):

El propósito es identificar y agrupar conforme a habilidades comunicativas destacadas a los estudiantes en distintos talleres de oratoria, creación literaria, prensa escrita, radio, escritura creativa, de libros ilustrados, libros electrónicos, libro-álbum, juego de rol, poesía, fan-fiction, etc. Estos talleres se conforman partir de la experiencia de la primera fase. La idea es aprovechar los "talentos" detectados por los facilitadores de la primera etapa al mismo tiempo que se involucra a los actoresestudiantes en el desarrollo de la conciencia social en su comunidad. La metodología de trabajo es a partir de proyectos en los cuales el alumno o los alumnos en coordinación con el asesor plantearán el producto a trabajar durante el semestre y establecerán la evidencia a lograr.

c) Fase V y VI, talleres de transposición de conocimientos (Comparte y Compartidos):

El propósito fue brindar elementos de intervención social que permitan al estudiante la transposición de sus conocimientos a través de la vertiente Sale y Valee, en la cual se trabaja en la potenciación del dominio del idioma español con alumnos de distintos niveles y modalidades educativas a 
partir de la lectura literaria. Para cada nivel de la vertiente Expresa se diseña un taller orientado al logro de las habilidades propuestas y evaluadas por PISA.

\section{Sale y Valee (vertiente externa)}

La vertiente externa es conocida como Sale y Valee, en ella se busca la formación lectora de alumnos de educación básica (preescolar, primaria y secundaria) y bachillerato de la Región de los Valles, a través de la trasposición de la metodología vivenciada durante la vertiente interna (Expresa) a través de servicio social de los estudiantes de CUValles. El aspecto de la lectura en la escuela debe abordarse desde el placer. Fomentar el gusto por la lectura literaria de esta manera para formar lectores. Debemos comenzar por despertar la lectura como una alternativa de ocio para los niños y jóvenes en México. No podemos atacar problemas de comprensión lectora si antes no trabajamos la parte de la lectura por placer. La estrategia consiste en talleres en las áreas de lectura y literatura infantil, bases pedagógicas y manejos de grupos.

a) Lectura y literatura infantil: conocimiento, manejo y uso de cuentos para niños, técnicas de manejo de voz y técnicas cuentacuentos.

b) Bases pedagógicas: aprendizaje significativo, teoría de las inteligencias múltiples, cuatro pilares de la educación, metacognición y juego en clase.

c) Manejo de grupos: técnicas colaborativas, estrategias de integración, juegos y actividades para ambientes colaborativos.

El trayecto formativo contempla una duración de 480 horas y los talleres de capacitación para iniciar la intervención en las instituciones educativas se realizarán al inicio del semestre con una duración de 12 horas. Además de las horas que el estudiante asista a la institución educativa, tiene que acudir a talleres de retroalimentación una vez por semana, cada uno con una duración de dos horas. Los talleres de retroalimentación son impartidos en las sub-sedes ubicadas a lo largo de los 14 municipios que conforman la región de los Valles.

\section{Informe de resultados Expresa - Sale y Valee}

Los alumnos de CUValles y escuelas/estudiantes de la región Valles que fueron beneficiados pertenecen a los calendarios del 2011B al 2013A.

Sobre el Informe de resultados del Expresa tenemos que en el calendario 2011B se apoyaron 593 estudiantes; en 2012A, 1 509; en 2012B, 1224 y finalmente, en 2013A a 2101 estudiantes.

El total de alumnos atendidos a partir del ciclo escolar 2011B hasta 2012B es de 5427 . El dato anterior contempla la cantidad total de alumnos inscritos en cada una de las diferentes fases del programa, lo cual no es indicativo de la cantidad de estudiantes atendidos a lo largo de su propio proceso.

En relación con la vertiente externa Sale y Valee, se presenta a continuación la relación de municipios, escuelas y alumnos atendidos y beneficiados con el proyecto en dos periodos: 
Los programas de investigación, promoción e intervención social para la mejora de habilidfades de lectocomprensión en la Región Ameca Jalisco en CUValles, Universidad de Guadalajara

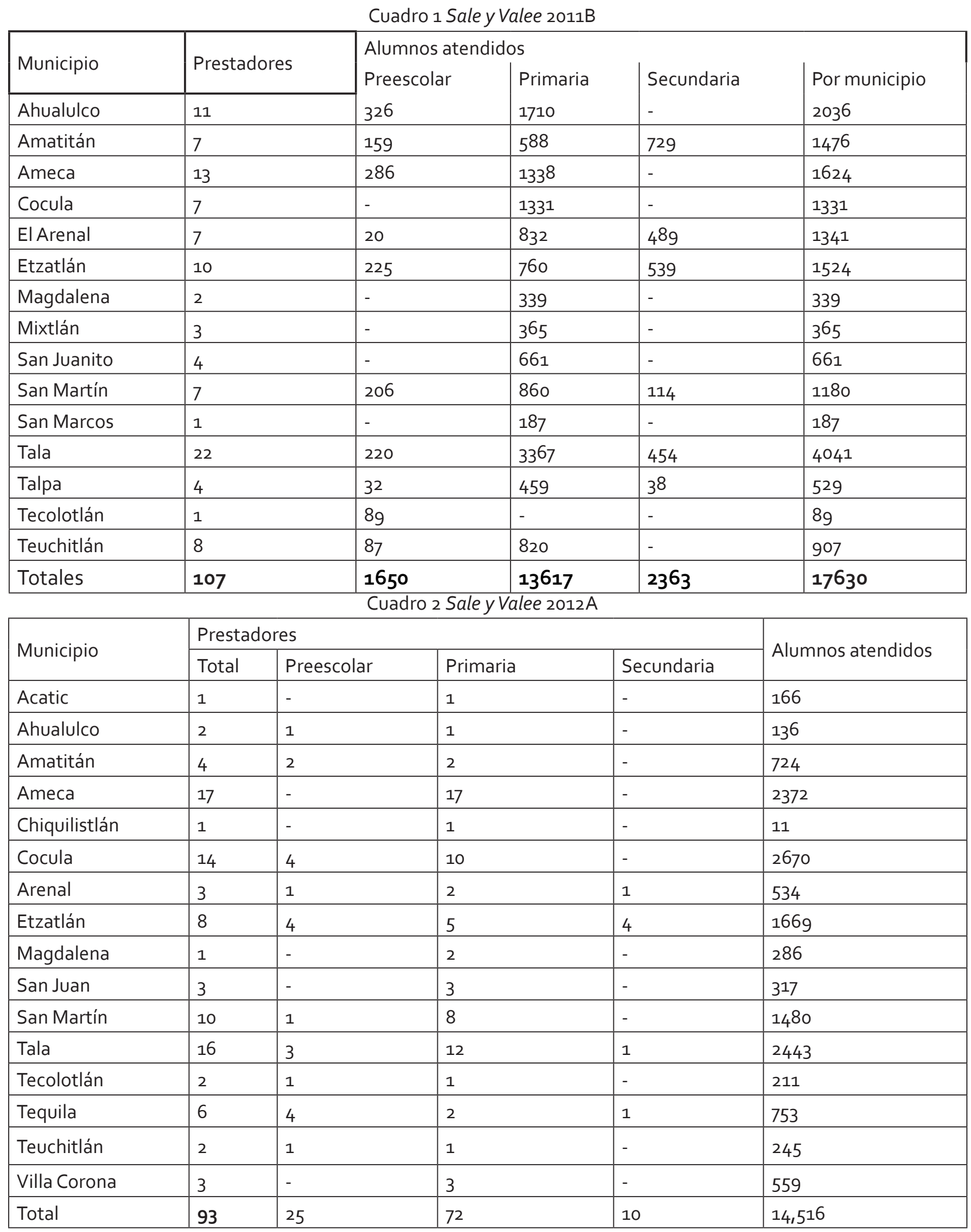


Mónica Almeida López, Rachel Garcíaeres, Reynaga, Hilde Aquino, Teresa Orozco. Colaboradores: Pablo Silva Villalever y Víctor Montero Gaytán

En total se atendieron 16 municipios con 106 estudiantes universitarios como parte de su servicio social. El total de alumnos de educación básica atendidos tanto de preescolar, primaria y secundaria fue de 17630 durante el semestre de agosto a diciembre de 2011 y de 14516 en el calendario 2012A con el apoyo de 93 prestadores en 117 escuelas ( 25 preescolares, 72 primarias y 10 secundarias de 18 municipios).

A partir de algunos instrumentos aplicados tanto a prestadores universitarios como a docentes, directivos y alumnos de las escuelas intervenidas, se crea la propuesta de formar parejas de trabajo en las escuelas intervenidas, lo cual implica que sean dos prestadores de servicio quienes asistan a las escuelas. Lo anterior con el propósito de crear un acompañamiento y un equipo de trabajo en donde se retroalimenten uno al otro. Esta nueva situación provocó una reducción casi a la mitad de las escuelas atendidas, pero una mejora en la calidad de las intervenciones en las mismas.

\section{Cuadro 3. Sale y Valee 2012B}

\begin{tabular}{|c|c|c|c|c|c|}
\hline \multirow{2}{*}{ Municipio } & \multicolumn{4}{|c|}{ Prestadores } & \multirow{2}{*}{$\begin{array}{l}\text { Alumnos } \\
\text { atendidos }\end{array}$} \\
\hline & Total & Preescolar & Primaria & Secundaria & \\
\hline Acatic & 1 & - & 1 & - & 231 \\
\hline Acatlán & 1 & - & 1 & - & 229 \\
\hline Ahualulco & 4 & - & 2 & - & 265 \\
\hline Amatitán & 5 & 1 & 1 & - & 378 \\
\hline Ameca & 18 & 6 & 7 & - & 1219 \\
\hline Cocula & 2 & - & 1 & - & 88 \\
\hline Arenal & 4 & 1 & 2 & - & 719 \\
\hline Etzatlán & 3 & 1 & - & 2 & 613 \\
\hline Guauch & 2 & - & 1 & - & 145 \\
\hline Magdalena & 1 & - & 2 & - & 285 \\
\hline San Juan & 1 & - & 1 & - & 40 \\
\hline San Marcos & 3 & - & 1 & - & 189 \\
\hline San Martín & 4 & 1 & 2 & - & 398 \\
\hline Tala & 8 & 1 & 4 & - & 900 \\
\hline Talpa & 2 & - & 2 & - & 239 \\
\hline Tequila & 2 & 1 & 1 & - & 383 \\
\hline Teuchitlán & 2 & 1 & - & 1 & 139 \\
\hline V. Corona & 2 & - & 2 & - & 402 \\
\hline Total & 64 & 13 & 30 & 3 & 6,862 \\
\hline
\end{tabular}

Fuente propia. 
Los programas de investigación, promoción e intervención social para la mejora de habilidfades de lectocomprensión en la Región Ameca Jalisco en CUValles, Universidad de Guadalajara

Uno de los principales retos de este proyecto es lograr la vinculación de la experiencia de servicio social con su trabajo de titulación. Pretendemos que al vivenciar en primera persona las necesidades de su comunidad, surjan problemáticas que den pie a proyectos de investigación que resuelvan las problemáticas reales de personas reales en su propia comunidad. Y esto sirva para tomar conciencia sobre su realidad, posicionarlos como líderes en su propia comunidad y obtener el grado de licenciatura en nuestra Universidad de Guadalajara.

Con respecto a las mejorías en las habilidades de los alumnos de educación básica se encontró que los profesores detectaron los siguientes cambios según encuesta aplicada en el calendario 2012 B.

Encuesta de salida realizada por universitarios capacitadores a profesores de educación básica para reconocer el impacto del programa de investigación hacia la comunidad.

La habilidad que más destaca es la de trabajar en grupo o equipo. En seguida se encuentra la facilidad de explicar lo que leen. La peor clasificada fue la de la utilización de un lenguaje más complejo, la cual también es una habilidad mucho más difícil de desarrollar, por lo que es razonable que sea así. Aun así encontramos que 34\% afirma sí encontrar un aumento en la complejidad en los rangos de casi siempre a siempre, y $44 \%$ en el de a veces. Los cuales indican mejoras sustantivas.

La investigación-acción es un proceso que sigue una evolución sistemática y cambia tanto al investigador como a las situaciones en las que éste actúa. Algunas ventajas de implicarse en procesos de investigación-acción se relacionan con un aumento de la autoestima profesional, la disminución del aislamiento profesional y el refuerzo de la motivación profesional. Permite que los profesionales investiguen y que se forme un profesional reflexivo.

\section{Referencias}

Delors, Jacques et al. (1996). La educación encierra un tesoro. Madrid: Santillana, Ediciones UNESCO.

DeSeCo:http://www.deseco.admin.ch/bfs/deseco/en/index/03/02.parsys. 78532.downloadList.94248.DownloadFile.tmp/2005.dscexecutivesummary.sp.pdf

RIEB http://basica.sep.gob.mx/reformaintegral/sitio/pdf/secundaria/plan/PlanEstudios2006.pdf

OCDE (s/a) El programa PISA de la OCDE. Qué es y para qué sirve, México, OCDE. Consultado en: http://www.oecd.org/dataoecd/58/51/39730818.pdf

INEE (2010) México en PISA 2009. México: INEE.

http://www.inee.edu.mx/BuscadorDocs/detallePub.action;jsessionid=BBBA9657E557AE90B4F A7047E44B0CDE?clave $=$ INEE-201102297

Freire, Paulo (1971). Sobre la acción cultural. Santiago de Chile: ICIRA. (1990). La naturaleza política de la educación. Cultura, poder y liberación. Madrid: Paidós. (2001). Pedagogía de la indignación. Madrid: Morata. (2008). La importancia de leer y el proceso de liberación. México: Siglo XXI. 
Mónica Almeida López, Rachel Garcíaeres, Reynaga, Hilde Aquino, Teresa Orozco. Colaboradores: Pablo Silva Villalever y Víctor Montero Gaytán

Lankshear, Colin y Michele Knobel (2008). Nuevos alfabetismos. Su práctica y el aprendizaje en el aula. Madrid: Morata.

Maturana, Humberto (2001). Emociones y lenguaje en educación y política. Santiago de Chile: Dolmen Ensayo.

y Francisco Varela (1990). El árbol del conocimiento. Las bases sociales del conocimiento humano. Madrid: Debate.

Morin, Edgar (1997). Introducción al pensamiento complejo. Barcelona: Gedisa. (1999). La cabeza bien puesta. Buenos Aires: Nueva Visión. (2002). Los siete saberes necesarios para la educación del futuro. Buenos Aires: Nueva Visión.

Perrenoud, Philippe (2010). Construir competencias desde la escuela. México: J. C. Sáenz.

Rimari Arias, Wilfredo (2011). "La innovación educativa”. Consultado en: www.fondep.gob.pe/ boletin/Innovacion.pdf consultado enero de 2011.

Schmelkes, S. (2001). La investigación en la innovación educativa. México: CINVESTAV.

Recibido: 18/05/15

Dictaminado: 12/10/15

Corregido: 05/11/15

Aceptado: 09/11/15 